\title{
Management of hepatic granulomatous tuberculosis complicating intravesical BCG for superficial bladder cancer
}

\author{
Vincent Fradet, MD, FRCSC; ${ }^{*}$ Christiane Gaudreau, MD; ${ }^{\dagger}$ Paul Perrotte, MD; ${ }^{*}$ Jean Côté, MD; ${ }^{+}$ \\ Jean-Marie Paquin, MD*
}

\begin{abstract}
Intravesical bacille Calmette-Guérin (BCG) therapy is the most effective treatment for high-risk superficial bladder cancer. Severe systemic complications are rare, but may occur in approximately $1 \%$ of cases. We report a severe complication of intravesical BCG: a disseminated Mycobacterium bovis infection with biopsy-proven granulomatous hepatitis in a patient with bladder cancer. We also elaborate on the different management alternatives.
\end{abstract}

CUAJ 2007;1(3):269-72

\section{Introduction}

Mycobacterium bovis was identified in 1904 by Nocard and it is the cause of bovine tuberculosis. Bacille Calmette-Guérin (BCG) vaccine, developed by Calmette and Guérin at Institut Pasteur (Lille, France), is a live-attenuated Mycobacterium bovis strain. Many therapeutic uses of the species have been described. Morales, in 1976, first described a technique of non-specific anti-cancer immunotherapy by bladder instillations of BCG for the treatment of superficial bladder cancer. ${ }^{1}$ BCG is the first choice of adjuvant treatment for recurrent or high-grade superficial bladder cancer. ${ }^{2,3}$ Complications related to its use have been well described, ${ }^{4}$ but serious parenchymatous infectious complications are not widely reported. We report the case of a disseminated Mycobacterium bovis BCG infection with biopsy-proven granulomatous hepatitis. We also present a review of literature and the management options.

\section{Case}

A 75-year-old otherwise healthy patient was treated for recurrent superficial bladder cancer. He underwent multiple trans-urethral resections (TURs) of high-grade T1 bladder tumours. He also received 2 induction courses of intravesical BCG therapy once a week for 3 weeks, and then subsequently for an additional 6 weeks. Despite this intensive management, the patient presented with tumour progression at the subsequent TUR. The tumour was found to be muscle invasive (stage T2). The metastatic workup, including an abdominopelvic CT scan, an endovenous pyelogram, a chest radiograph, a complete blood count and biochemical laboratories, was negative with the exception of elevated liver enzymes (aspartate aminotransferase max $68 \mathrm{U} / \mathrm{L}$, alanine aminotransferase max $59 \mathrm{U} / \mathrm{L}$, alkaline phosphatase max $210 \mathrm{U} / \mathrm{L}$ ). A preopera- tive hepatology consultation was ordered and a transjugular hepatic biopsy revealed a normal portocaval gradient. Histology demonstrated granulomas; however, auramine coloration for acid-fast bacteria, Grocott coloration for fungi and periodic acid-Schiff staining for other microorganisms were all negative. The purified protein derivate (PPD) subcutaneous test was negative. The patient underwent radical cystectomy, which was uneventful. The final pathology demonstrated muscle invasive transitional cell carcinoma (stage T2). At the time of surgery, a planned liver biopsy was done. Histology demonstrated granulomatous hepatitis and liver tissue culture, Mycobacterium infection.

The patient demonstrated persistent mild fever in the postoperative period as well as persistent intra-abdominal bleeding. Initial bacterial, viral and fungal cultures were all negative. Because the patient's infectious state was progressing, an empiric triple antituberculous therapy was started, with isoniazide $300 \mathrm{mg}$, rifampin $600 \mathrm{mg}$ and ethambutol $1200 \mathrm{mg}$, and it was complemented with pyridoxine (B6 vitamin) $50 \mathrm{mg}$ daily. A Mycobacterium was isolated in 2 out of 3 Midget broth (BD Bactec, Franklin Lakes, NJ) preoperatively taken urine cultures after 11 days of incubation, and the polymerase chain reaction (PCR) for group tuberculosis on the Mycobacterium strain was positive. The final identification, done by mycolic acid chromatography at the Quebec reference laboratory, Laboratoire de Santé Publique du Québec, reported a Mycobacterium bovis BCG susceptible to isoniazide, rifampin and ethambutol but, as expected, resistant to pyrazinamide. Two blood cultures using Myco F Lytic (BD Bactec, Franklin Lakes, NJ) that were taken before the start of empiric therapy were also positive for the same 
Mycobacterium after 27 days of incubation. The intra-operative hepatic biopsy was cultured. It initially demonstrated an absence of alcohol-resistant bacille and a negative PCR for mycobacteria, but after 25 days the culture became positive for the same Mycobacterium bovis BCG.

The patient unfortunately experienced numerous complications, including renal failure, and he eventually died of respiratory insufficiency.

\section{Discussion}

We report a disseminated Mycobacterium bovis BCG infection with granulomatous hepatitis after bladder BCG instillations for bladder cancer. In their series, Debois and colleagues, ${ }^{5}$ and Lamm and colleagues $^{4,6}$ reported a $45 \%-90 \%$ rate of irritative symptoms and a $35 \%-43 \%$ rate of hematuria. Lowgrade fever was reported in $16 \%-28 \%$ of cases, while high-grade fever was reported in $2 \%-4.2 \%$. Serious complications related to intravesical BCG has an estimated rate of $0.35 \%-1.9 \%$, the vast majority $(70 \%-75 \%)$ of those being systemic. Debois and collegues analyzed the pharmacovigilance French data and described an infectious cause of serious BCG complications in $63 \%$ of cases, while the other causes were rheumatologic and unexpected. Lamm and colleagues ${ }^{4}$ reviewed the existing data in 1986 , in which $78 \%$ of serious complications were attributed to infectious causes (including fever above $103^{\circ} \mathrm{F}$ as infectious cause). In both series, diagnosis of infection, sometimes without any proof, was assumed. This implies that the true incidence of infectious complications related to intravesical BCG is probably less than what is estimated in the literature. Disseminated miliary disease, ${ }^{7}$ vertebral osteomyelitis, ${ }^{8}$ mycotic aneurysm ${ }^{9}$ and skin abscess ${ }^{10}$ have also been reported. The death rate related to intravesical BCG use has never been calculated, but it is estimated to be very low; there were 9 deaths (related or not) in Debois and collagues' pharmacovigilance study covering 3 years and an unknown number of treated patients. Acute BCG-related infectious complications were the cause of death in 4 patients on whom information was detailed. No deaths were reported in Lamm and colleagues' series.

Based on histological diagnoses from liver biopsies, the liver has been reported as a potential site of disseminated disease, ${ }^{11}$ but tissue culture of Mycobacterium bovis BCG, which is the ultimate proof of the disease after intravesical BCG has not been described in the literature. Lamm and colleagues ${ }^{4,6}$ reported a combined incidence of pneumonitis and hepatitis of $0.9 \%$, but 7 out of 46 $(15 \%)$ severe systemic complications had indirect proof of disseminated BCG with granulomatous lesions on biopsy and were considered diagnostic. ${ }^{6}$ Granulomatous hepatitis is not a specific diagnosis. Differential diagnosis also includes sarcoidosis, foreign body granuloma (e.g., in intraveinous powder drug users) and mycotic infection such as histoplasmosis or toxoplasmosis. Histologically, mycobacterial granulomas are normally located around the centrolobular vein, have a more significant caseification reaction (but not in immunosuppressed patients) and have a predominantly lymphocytic inflammatory infiltrate in addition to well-formed epithelioid granulomas, caseous necrosis, and an absence of Langhans giant cells. ${ }^{12}$ Clinically, fever and malaise are the predominant manifestations. The liver can be enlarged and painful upon palpation. Hepatocellular enzymes are usually elevated, but sometimes only hyperbilirubinemia and elevated alkaline phosphatase are noted in the laboratory workup. The syndrome of inappropriate anti-diuretic hormone secretion $(\mathrm{SIADH})$ has also been described.

\section{Suggested management}

Prevention is the most important management strategy. Recent surgery (2-4 weeks after TUR), traumatic catheterization or cystoscopy, active hematuria, immunocompromised status (i.e., transplant, leukemia, Hodgkin's disease or AIDS), fever of unknown origin, pregnancy and active urinary tract infection are all contraindications to the administration of intravesical BCG and mandate postponing the treatment. Strict surveillance of patients before treating them with intravesical BCG is of the utmost importance, and the severity of symptoms at the beginning of treatment is predictive of the necessity to stop the maintenance BCG treatment. ${ }^{13,14}$ In most of the reported cases with severe BCG-related infectious complications, a traumatic catheter or another contraindication is described. Prophylactic isoniazide has been evaluated during intravesical BCG therapy in a randomized study and is not considered efficacious owing to similar incidence of short-term complications and adverse effects related to isoniazide. ${ }^{15}$ 
If systemic complications from BCG are suspected, the following mycobacterial cultures are suggested: 3 urine cultures, 2 blood cultures and a liver biopsy for culture and histology, if hepatic granulomatous disease is suspected. Broth cultures are much more sensitive for Mycobacterium than are solid media. M. bovis BCG is much more fastidious to isolate in broth or on solid media than Mycobacterium tuberculosis or Mycobacterium other than the tuberculosis group. PCR, can also be performed on tissue or urine (but not on blood) to diagnose the Mycobacterium tuberculosis group, which encompasses Mycobacterium bovis. Having a definitive diagnosis, by liquid chromatography or by $16 \mathrm{~S}$ rRNA gene sequencing, will help tailor the intensity of the therapy. Sensitivity testing to different antimicrobial agents also helps to rule out resistant strains.

Mycobacterium bovis BCG and M. bovis are intrinsically resistant to pyrazinamide. Resistance to $\beta$-lactams, macrolides (except clarithromycin) and some aminoglycosides has recently been reported..$^{16}$ The suggested first-line antimicrobial treatment for M. bovis BCG is isoniazide $300 \mathrm{mg}$ daily and rifampin $600 \mathrm{mg}$ daily with or without ethambutol $1200 \mathrm{mg}$ daily by mouth for 6 months. ${ }^{17}$ Ethambutol is recommended as complementary treatment for patients with significant systemic disease or to replace isoniazide in patients who do not tolerate the drug owing to adverse effects (i.e., liver dysfunction, fever, malaise or maculopapular eruption). Aluminum-containing antacids reduce oral absorption of isoniazide. Monotherapy is not recommended because of the development of resistance. Other alternatives include the addition of ofloxacin $400 \mathrm{mg}$ daily, doxycyclin and gentamicin. ${ }^{16,18}$ The usefulness of cycloserine is controversial. Because it inhibits BCG growth rapidly (24 h compared with isoniazide, which may take as long as 1 week), some consider cycloserine potentially life-saving in patients with fulminant systemic infectious complications from BCG. However, cycloserine has severe central nervous system side effects and $M$. Bovis BCG resistance has been frequently reported. ${ }^{16,19}$ Cycloserine should not be used routinely. The actual recommended length of treatment is 6 months for those patients manifesting extravesical symptoms, but some authors prefer treating up to 12 months for culture-proven extravesical tissue disease. ${ }^{8}$
Prednisolone $40 \mathrm{mg}$ daily may be considered for patients with systemic disease as hypersensitivity reaction is initially difficult to differentiate from infectious reaction. ${ }^{20}$ If prescribed, corticosteroids should be maintained as long as systemic symptoms are present. They should be gradually tapered off because exacerbation of this hypersensitivity response has been seen in patients who have stopped corticosteroids abruptly. ${ }^{3}$ Pyridoxine 10-50 mg daily should be added to prevent some side effects of isoniazid, like peripheral neuropathy, especially in high-risk patients such as the elderly, diabetics, people with chronic liver dysfunction and HIV patients.

Although the immunological mechanism of intravesical BCG against bladder cancer is becoming better understood, ${ }^{21}$ many unanswered questions remain. Long-term mycobacterial persistence after intravesical BCG has been proven and represents an infectious risk but also a possible explanation of immune anticancer effect. ${ }^{22}$ What should the waiting time between suspected mycobacterial infection after BCG and radical treatment of a muscle invasive bladder cancer be? Should all patients have empirical treatment before undergoing radical surgery?

In conclusion, granulomatous parenchymatous disease is a rare but potentially life-threatening complication of intravesical BCG and it is often associated with systemic febrile disease. Specific mycobacterial cultures and diagnostic biopsy should be part of the standard management. Appropriate treatment should be initiated rapidly because death from septic complications can happen. Collaboration between urologist, infectious disease specialist, pathologist and hepatologist is essential for these complicated cases.

From the *Departement of Surgery (urology), the †Department of Microbiology and the łDepartment of Pathology, Centre Hospitalier de I'Université de Montréal-Hôpital St-Luc, Montréal, Que.

This article has been peer reviewed.

Competing interests: None declared.

\section{References}

1. Morales A, Eidinger D, Bruce AW. Intracavitary bacillus Calmette-Guerin in the treatment of superficial bladder tumors. J Urol 1976;116:180.

2. Kamat AM, Lamm DL. Immunotherapy for bladder cancer. Curr Urol Rep 2001;2:62-9.

3. Lamm DL. Efficacy and safety of bacillus Calmette-Guerin immunotherapy in superficial bladder cancer. Clin Infect Dis 2000;31 (Suppl. 3):86-90. 
4. Lamm DL, Stogdill VD, Stogdill BJ, et al. Complications of bacillus Calmette-Guerin immunotherapy in 1,278 patients with bladder cancer. J Urol 1986;135:272-4.

5. Debois H, Loupi E, Saliou P, et al. Evaluation de la tolerance de la BCG thérapie endovéscale en France: analyse des événements indésirables graves notifiés sur une période de trois ans. Progrès en Urol 2001;1 1:458-65.

6. Lamm DL, van der Meijden PM, Morales A, et al. Incidence and treatment of complications of bacillus Calmette-Guérin intravesical therapy in superficial bladder cancer. J Urol 1992;147:596-600.

7. Elkabani M, Greene JN, Vincent AL, et al. Disseminated Mycobacterium bovis after intravesicular bacillus Calmette-Guérin treatments for bladder cancer. Cancer Control 2000;7:476-80.

8. Aljada IS, Crane JK, Corriere N, et al. Mycobacterium bovis BCG causing vertebral osteomyelitis (Pott's Disease) following intravesical BCG therapy. J Clin Microbiol 1999; 37:2106-8.

9. Kamphuis JT, Buiting AG, Misere JF, et al. BCG immunotherapy: be cautious of granulomas. Disseminated BCG infection and mycotic aneurysm as late complication of intravesical BCG instillations. Neth J Med 2001;58:71-5.

10. Mangiarotti B, Trinchieri A, Marconato R, et al. Skin abscess after intravesical instillation of bacillus Calmette-Guerin for prophylactic treatment of transitional cell carcinoma. J Urol 2002;168:1094-5.

11. Göttke MU, Wong P, Muhn C, et al. Hepatitis in disseminated bacillus CalmetteGuérin infection. Can I Gastroenterol 2000;14:333-6.

12. Al-Bhlal LA. Pathologic findings for bacille Calmette-Guérin infections in immunocompetent and immunocompromised patients. Am I Clin Pathol 2000;113:703-8.

13. Saint F, Irani J, Patard JJ, et al. Tolerability of bacille Calmette-Guerin maintenance therapy for superficial bladder cancer. Urology 2001;57:883-8.
14. Saint F, Irani J, Salomon L, et al. Etude de la tolérance et de l'efficacité des instillations endovésicales de Bacille Calmette-Guérin dans le traitement prophylactique des tumeurs superficielles de vessie, en utilisant un traitement d'entretien. Progrès Urol 2001;11:647-56.

15. Vegt PDJ, van der Meiiden APM, Sylvester R, et al. Does isoniazid reduce side effects of intravesical bacillus Calmette-Guerin therapy in superficial bladder cancer? Interim Results of European Organization for Research and Treatment of Cancer Protocol 30911. J Urol 1997;157:1246-9.

16. Durek C, Rusch-Gerdes $S$, Jocham D, et al. Sensitivity of BCG to modern antibiotics. Eur Urol 2000;37 (Suppl 1):21-5.

17. Huang TC. Management of complications of bacillus Calmette-Guerin immunotherapy in the treatment of bladder cancer. Ann Pharmacother 2000;34:529-32.

18. Fendler JP, Debois H, Mège JL, et al. BCGite: I'ofloxacine peut-elle constituer une alternative au traitement antituberculeux? Progrès Urol 2002;12:309-12.

19. Su WJ, Huang $C Y$, Huang $C Y$, et al. Utility of PCR assays for rapid diagnosis of $B C G$ infection in children. Int I Tuberc Lung Dis 2001;5:380-4.

20. Grange JM. Complications of bacilli Calmette-Guérin (BCG) vaccine. Commun Dis Public Health 1998;1:84-8.

21. Alexandroff $A B$, Jackson $A M, O^{\prime} D$ Donnell $M A$, et al. $B C G$ immunotherapy of bladder cancer: 20 years on. Lancet 1999;353:1689-94.

22. Durek C, Richter E, Bastek A, et al. The fate of bacillus Calmette-Guerin after intravesical instillation. J Urol 2001;165:1765-8.

Correspondence: Dr. Vinceny Fradet, Clinique d'Urologie Berger, 416-1000 chemin Sainte-Foy, Québec QC GIS 2L6; vincent.fradet@videotron.ca 